\title{
Repercussões da COVID-19 para as gestantes
}

\author{
Repercussions of COVID-19 for pregnant women
}

Repercusiones de COVID-19 para mujeres embarazadas

Recebido: 17/12/2020 | Revisado: 24/12/2020 | Aceito: 28/12/2020 | Publicado: 02/01/2021

Aclênia Maria Nascimento Ribeiro

ORCID: https://orcid.org/0000-0002-5582-9663 Universidade Federal do Piauí, Brasil

E-mail: aclennya@hotmail.com

Gabriela Oliveira Parentes da Costa

ORCID: https://orcid.org/0000-0001-9473-8986 Instituto Federal de Educação, Ciência e Tecnologia do Maranhão, Brasil

E-mail: gabiparents@ hotmail.com

Lânia da Silva Cardoso

ORCID: https://orcid.org/0000-0003-1612-3147 Instituto de Ensino Superior Múltiplo, Brasil

E-mail: lanniia@gmail.com

Diana Nogueira Villa Jatobá

ORCID: https://orcid.org/0000-0003-4346-8335

Centro Universitário UNINOVAFAPI, Brasil

E-mail: devilla@ hotmail.com

Mara Cléssia de Oliveira Castro

ORCID: https://orcid.org/0000-0002-1808-7963

Centro Universitário UNINOVAFAPI, Brasil

E-mail: maraclessia@hotmail.com

Luzia Fernandes Dias

ORCID: https://orcid.org/0000-0003-4770-2782

Centro Universitário Maurício de Nassau, Brasil

E-mail: estrela.lu@hotmail.com

Felipe de Sousa Moreiras

ORCID: https://orcid.org/0000-0002-8703-1429

Universidade Federal do Piauí, Brasil

E-mail: felipe_moreiras007@hotmail.com

Yara Maria Rêgo Leite

ORCID: https://orcid.org/0000-0002-4868-2624 Hospital Universitário da Universidade Federal do Piauí, Brasil

E-mail: yara_leite189@ hotmail.com

Ricardo Clayton Silva Jansen

ORCID: https://orcid.org/0000-0002-6392-8100

Universidade Federal do Maranhão, Brasil

E-mail: ricardojansen_20@hotmail.com

Juliana de Menezes Dantas

ORCID: https://orcid.org/0000-0002-6085-4361

Hospital Universitário da Universidade Federal do Piaú, Brasil

E-mail: juldantas_pi@hotmail.com

Maria Tamires Alves Ferreira

ORCID: https://orcid.org/0000-0001-7822-9575

Faculdade Estácio de Teresina, Brasil

E-mail: thammyaf@hotmail.com

Carolina Silva Vale

ORCID: https://orcid.org/0000-0001-8269-9705

Hospital Universitário da Universidade Federal do Piauí, Brasil

E-mail: carol.vale.enf@hotmail.com

Fernanda Mendes Dantas e Silva

ORCID: https://orcid.org/0000-0003-3084-3263

Universidade Federal do Piauí, Brasil

E-mail: fernandantass@gmail.com

Evellyn Stefanne Bastos Marques

ORCID: https://orcid.org/0000-0002-8325-6188

Universidade Federal do Piauí, Brasil

E-mail: evellynbastos15@gmail.com

Dallyane Cristhefane Carvalho Pinto

ORCID: https://orcid.org/0000-0002-8212-4765

Centro Universitário UNINOVAFAPI, Brasil

E-mail: dallyanemonaco@hotmail.com 
Luciana Stanford Baldoino

ORCID: https://orcid.org/0000-0002-5052-7607 Instituto de Ensino Superior Múltiplo, Brasil E-mail: 1sbaldoino@hotmail.com

Maria Paula da Silva Oliveira

ORCID: https://orcid.org/0000-0003-3831-5430 Centro Universitário UNINOVAFAPI, Brasil E-mail: paulinha-oliveira08@hotmail.com

\begin{abstract}
Resumo
Objetivo: Refletir acerca das repercussões da COVID-19 para as gestantes. Metodologia: Trata-se de uma revisão integrativa da literatura realizada nas bases de dados: MEDLINE, SCIELO e LILACS. Os critérios de inclusão foram: artigos disponíveis com as mais variadas metodologias, nos idiomas português, inglês ou espanhol, publicados a partir do ano de 2019 e que tratassem especificamente da COVID-19 no contexto da gravidez e suas repercussões para esse público. Foram excluídos artigos duplicados e que não se adequaram aos objetivos do estudo. Assim, após a aplicação dos critérios de inclusão e exclusão, a amostra final constituiu-se de 8 estudos. Resultados e discussão: A análise dos estudos selecionados permitiu inferir que existem muitas controvérsias em relação aos cuidados com as gestantes, principalmente quanto ao cenário trabalho de parto de uma mãe diagnosticada com COVID-19, repercutindo assim em um contexto privado de acompanhante e do contato direto com o recém-nascido em algumas instituições de saúde que infligem esses direitos das gestantes. Em relação à ansiedade, estresse e depressão, os resultados relacionaram o isolamento social causado pela pandemia, como um fator desencadeante de tais problemas psicológicos. Já, as complicações gestacionais causadas pela COVID-19 ainda estão sendo esclarecidas, a medida em que vão surgindo novos casos e estudos. Considerações finais: Assim, enfatiza-se a importância dos profissionais da saúde, no tocante as orientações prestadas a gestante e seus familiares, como forma de prevenir a infecção, desmitificar mitos, esclarecendo dúvidas que possam contribuir para diminuir a sobrecarga de ansiedade durante gestação.
\end{abstract}

Palavras-chave: Gestantes; Análise de consequências; COVID-19; Infecções por coronavírus.

\begin{abstract}
Objective: To reflect on the repercussions of COVID-19 for pregnant women. Methodology: This is an integrative literature review carried out in the databases: MEDLINE, SCIELO and LILACS. The inclusion criteria were: articles available with the most varied methodologies, in Portuguese, English or Spanish, published from 2019 and specifically dealing with COVID-19 in the context of pregnancy and its repercussions for this audience. Duplicate articles that did not fit the study objectives were excluded. Thus, after applying the inclusion and exclusion criteria, the final sample consisted of 8 studies. Results and discussion: The analysis of the selected studies allowed us to infer that there are many controversies in relation to the care for pregnant women, especially regarding the labor scenario of a mother diagnosed with COVID-19, thus affecting a private companion and contact context directly with the newborn in some health institutions that inflict these rights of pregnant women. Regarding anxiety, stress and depression, the results related the social isolation caused by the pandemic, as a triggering factor for such psychological problems. The gestational complications caused by COVID-19 are still being clarified, as new cases and studies are emerging. Final considerations: Thus, the importance of health professionals is emphasized, with regard to the guidance provided to pregnant women and their families, as a way to prevent infection, demystify myths, clarifying doubts that may contribute to reduce the overload of anxiety during pregnancy.
\end{abstract}

Keywords: Pregnant women; Analysis of consequences; COVID-19; Coronavirus infections.

\title{
Resumen
}

Objetivo: Reflexionar sobre las repercusiones del COVID-19 en las mujeres embarazadas. Metodología: Se trata de una revisión integrativa de la literatura realizada en las bases de datos: MEDLINE, SCIELO y LILACS. Los criterios de inclusión fueron: artículos disponibles con las más variadas metodologías, en portugués, inglés o español, publicados a partir de 2019 y que aborden específicamente el COVID-19 en el contexto del embarazo y sus repercusiones para esta audiencia. Se excluyeron los artículos duplicados que no se ajustaban a los objetivos del estudio. Así, tras aplicar los criterios de inclusión y exclusión, la muestra final estuvo formada por 8 estudios. Resultados y discusión: El análisis de los estudios seleccionados permitió inferir que existen muchas controversias en relación a la atención a la gestante, especialmente en cuanto al escenario laboral de una madre diagnosticada de COVID-19, afectando así a un acompañante privado y contexto de contacto. directamente con el recién nacido en algunas instituciones de salud que infringen estos derechos de las mujeres embarazadas. En cuanto a la ansiedad, el estrés y la depresión, los resultados relacionaron el aislamiento social provocado por la pandemia, como factor desencadenante de dichos problemas psicológicos. Las complicaciones gestacionales causadas por COVID-19 aún se están aclarando, a medida que surgen nuevos casos y estudios. Consideraciones finales: Así, se enfatiza la importancia de los profesionales de la salud, en lo que respecta a la orientación brindada a las embarazadas y sus familias, como una forma de prevenir la infección, desmitificar mitos, aclarar dudas que puedan contribuir a reducir la sobrecarga de ansiedad durante el embarazo.

Palabras clave: Mujeres embarazadas; Análisis de consecuencias; COVID-19; Infecciones por coronavirus. 


\section{Introdução}

A Síndrome Respiratória Aguda Grave do Coronavírus 2 (SARS-CoV-2) é um novo tipo de coronavírus (COVID-19) que já atingiu quase todos os países do mundo, acometendo a humanidade sem distinção de raça, etnia, cultura ou classe social, agravando as condições daqueles que estão incluídos nos grupos de vulnerabilidades, dentre os quais a Organização Mundial da Saúde (OMS) classificou as gestantes em razão do elevado risco de morbimortalidade (SousaBastos, 2020; Zaigham \& Andersson, 2020).

Levando em consideração o ciclo gravídico-puerperal que é um período em a mulher sofre alterações profundas nos aspectos físicos, psíquicos e sociais, podendo surgir no decorrer da gravidez importantes modificações na sua personalidade, além de predispor a complicações por infecções respiratórias. Logo o cuidado com a gestante não deve sofrer descontinuidade que ocasione o aumento no número de comorbidades e agravos (Wagner et al., 2020).

No entanto, a infecção do Coronavírus se torna mais um motivo de preocupação entre as gestantes e familiares que enfrentam o medo, a ansiedade e a incerteza, expondo-se ao risco de contaminação, além da falta de informações seguras a respeito do vírus, a sensação de perda de controle na gravidez e no parto; e as perspectivas em relação ao amanhã (Souto et al., 2020).

Embora ainda não existem evidências se o vírus pode ser transmitido da mãe para o bebê durante a gravidez, nos últimos meses da gestação as mulheres podem ser seriamente afetadas por algumas infecções respiratórias e, portanto, é importante tomar precauções para prevenir efeitos adversos graves como pneumonia ou até mesmo a morte materna-infantil (Furlan et al., 2020).

Nesse contexto, é importante ressaltar que os profissionais da saúde devem assegurar, à mulher, o direito da atenção humanizada à gravidez, ao parto e ao puerpério, bem como à criança o direito ao nascimento seguro e ao crescimento e ao desenvolvimento saudáveis (Brasil, 2011). Assim, diante do momento histórico em que estamos vivenciando e das complicações do período gravídico, resolveu- se realizar o presente estudo com o objetivo de refletir acerca das repercussões da COVID-19 para as gestantes.

\section{Metodologia}

O estudo foi realizado seguindo o método de revisão integrativa da literatura que segundo Pereira et al. (2020), consiste em uma revisão narrativa da literatura, na qual é possível ter acesso a pesquisas científicas com o obtivo de favorecer a abordagem teórica acerca da temática selecionada.

A pesquisa foi realizada e direcionada pela seguinte questão norteadora: quais as repercussões da COVID-19 para as gestantes? Convém ressaltar que essa questão norteadora foi elaborada de acordo com a estratégia PICo (P - paciente; I interesse; Co - contexto), que segundo Santos, Pimenta, \& Nobre (2007), pode ser realizada para desenvolver questões de pesquisa de naturezas diversas, provenientes da clínica, do gerenciamento de recursos humanos e materiais, da busca de estratégias para avaliação de sintomas, entre outras.

Para responder a questão do estudo foi realizada uma coleta de dados no mês de dezembro de 2020 nas bases de dados: National Library of Medicine (MEDLINE); Scientific Electronic Library Online (SCIELO) e LILACS (Literatura Latino-Americana e do Caribe em Ciências da Saúde).

Para a localização dos estudos relevantes, que respondessem à pergunta de pesquisa, utilizou-se de descritores indexados nos idiomas português, inglês e espanhol. Os descritores foram obtidos a partir do Medical Subject Headings (MESH) e dos Descritores em Ciências da Saúde (DeCS) conforme o Quadro 1. 
Quadro 1 - Elementos da estratégia PICo e descritores utilizados. Timon -MA, Brasil, 2020.

\begin{tabular}{|c|c|c|}
\hline ELEMENTOS & MESH & DECS \\
\hline "Gestantes" & "Pregnant Women" & "Pregnant Women" \\
& & 'Mujeres Embarazadas" \\
\hline I & "Consequence" & "Análise de Consequências" \\
"Repercussões" & "Consequence Analysis" \\
"Pandemia da & "Coronavirus Infections" & "Infecções por Coronavírus" \\
COVID-19" & & "Coronavirus Infections" \\
"COVID-19" & & "Infecciones por Coronavirus" \\
\hline
\end{tabular}

Fonte: Autores.

Foram considerados como critérios de inclusão: artigos disponíveis com as mais variadas metodologias, nos idiomas português, inglês ou espanhol, publicados a partir do ano de 2019 e que tratassem especificamente da COVID-19 no contexto da gravidez e suas repercussões para esse público. Foram excluídos artigos duplicados e que não se adequaram aos objetivos do estudo. Assim, após a aplicação dos critérios de inclusão e exclusão, a totalidade dos artigos que fizeram parte da amostra final foi de 8 estudos.

Os artigos selecionados foram apresentados em quadros e discutidos de forma narrativa baseados na literatura disponível acerca da temática. Assim, no Quadro 2, observa-se as principais características dos artigos selecionados neste trabalho de revisão integrativa.

Quadro 2 - Característica dos estudos selecionados. Timon - MA, Brasil. 2020.

\begin{tabular}{|c|c|c|c|c|}
\hline $\mathbf{N}^{\circ}$ & Autor & Título & Ano & Delineamento \\
\hline 1 & Estrela et al. & $\begin{array}{l}\text { Gestantes no contexto da pandemia da COVID-19: } \\
\text { reflexões e desafios. }\end{array}$ & 2020 & Estudo descritivo \\
\hline 2 & $\begin{array}{l}\text { Muhidin, } \\
\text { Moghadam, } \\
\& \underline{\text { Vizheh }}\end{array}$ & $\begin{array}{l}\text { Analysis of Maternal Coronavirus } \text { Infections and } \\
\text { Neonates Born to Mothers with 2019-nCoV; a } \\
\text { Systematic Review }\end{array}$ & 2020 & $\begin{array}{l}\text { Revisão } \\
\text { sistemática }\end{array}$ \\
\hline 3 & Liu et al. & $\begin{array}{l}\text { Coronavirus disease } 2019 \text { (COVID-19) during } \\
\text { pregnancy: a case series }\end{array}$ & 2020 & Estudo de caso \\
\hline 4 & Zhu et al. & $\begin{array}{l}\text { Clinical analysis of } 10 \text { neonates born to mothers with } \\
\text { 2019-nCoV pneumonia. }\end{array}$ & 2020 & Estudo de caso \\
\hline 5 & Rondelli et al. & $\begin{array}{l}\text { Assistência às gestantes e recém-nascidos no contexto } \\
\text { da infecção COVID-19: uma revisão sistemática. }\end{array}$ & 2020 & $\begin{array}{l}\text { Revisão } \\
\text { sistemática }\end{array}$ \\
\hline 6 & Ferreira et al. & $\begin{array}{l}\text { Vida Fisicamente Ativa como Medida de Enfrentamento } \\
\text { ao COVID-19. }\end{array}$ & 2020 & Estudo descritivo \\
\hline 7 & Li et al. & Maternal and neonatal outcomes of pregnant women & 2020 & Estudo de caso- \\
\hline
\end{tabular}




\begin{tabular}{|l|l|l|l|l|}
\hline & & with COVID-19 pneumonia: a case-control study. & & controle \\
\hline 8 & Paz et al. & $\begin{array}{l}\text { Barriers imposed in the relationship between puerperal } \\
\text { women and newborns in the COVID-19 pandemic } \\
\text { scenario. }\end{array}$ & 2020 & Estudo descritivo \\
\hline
\end{tabular}

Fonte: Autores.

Ao analisar o Quadro 2, é possível inferir que todos os artigos que compuseram a amostra final foram publicados no ano de $2020(100 \%)$. Quanto ao delineamento dos estudos, prevaleceram aqueles que utilizaram como metodologia o estudo descritivo (37,5\%). No entanto, outros métodos também foram observados como a revisão sistemática (25\%), estudo de caso (25\%) e estudo de caso-controle $(12,5 \%)$.

\section{Resultados e Discussão}

Repentinamente, a doença causada pelo Coronavírus foi disseminada pelo mundo, com rapidez e letalidade, dizimando a população vulnerável e com comorbidades. Neste grupo de vulneráveis se encontram as gestantes, que diante da pandemia causada pela COVID-19 estiveram alertas e amedrontadas pela incerteza de possíveis complicações causadas pelo vírus (Estrela et al., 2020).

Para Clode \& Areia (2020), as grávidas são o grupo de maior fragilidade durante a pandemia e necessitam de proteção, bem como de orientações quanto aos riscos para elas. As orientações sobre as formas graves da doença e as medidas para proteção, principalmente, o distanciamento social, devem se estender a seus familiares, que precisam contribuir no cuidado da gestante.

Considerando as controvérsias entre as evidências para a impossibilidade ou não de gestantes e crianças desenvolverem quadros mais graves da doença (Brasil, 2020a; Unfpa, 2020), deve-se ter precaução especial com relação as formas de contaminação, como a transmissão vertical e amamentação (Brasil, 2020b; Sbp, 2020).

Com precaução, o Brasil incluiu as grávidas em todas as idades gestacionais, na última atualização do Protocolo de Manejo Clínico da Infecção da COVID-19, além disso, incluiu as puérperas com até duas semanas de pós parto, as mulheres que tiveram aborto ou perda fetal, também, como grupo com fatores de risco para complicações de infecção da COVID-19 e alertou para atenção especial a este grupo (Brasil, 2020c).

Estudos afirmam ainda, que devido as alterações metabólicas e imunológicas que ocorrem durante a gestação, essas mulheres devem ter prioridade na assistência à saúde (Rcog, 2020). Assim, existe a preocupação de que as gestantes, caso infectadas pelo SARS-CoV-2 desenvolvam formas graves da doença, e necessitem de suporte avançado de vida, em unidades de terapia semi-intensiva ou intensiva (Liu et al., 2020a; Zhu et al., 2020).

Rondelli et al. (2020) em seu estudo, afirmam que mesmo que existam algumas pesquisas que apontem poucas chances de complicações da infecção pela COVID-19 em gestantes, em contrapartida, há consequências graves para o feto e recém-nascido, especialmente, quando o contágio ocorre no terceiro trimestre da gestação, corroborando com as recomendações de órgãos competentes e outros estudos, que defendem o distanciamento social e remanejamento para funções administrativas, no caso daquelas que atuam como profissionais da saúde.

Em um estudo realizado por Muhidin, Moghadam, \& Vizheh (2020), foi verificado que febre baixa e tosse foram os principais sintomas relatados por mulheres grávidas. No entanto, observou-se como complicações pré-natais: sofrimento fetal, ruptura prematura de membranas e trabalho de parto prematuro. Foi verificado ainda que duas mulheres necessitaram de leitos de terapia intensiva e ventilação mecânica (VM), e uma delas apresentou disfunção de vários órgãos e utilizou oxigenação por 
membrana extracorpórea (ECMO). Todavia, é importante dizer que nesse mesmo estudo não houve casos de morte em gestantes.

Ainda em relação aos sintomas da COVID-19 nas gestantes, Juan et al. (2020), notou em sua pesquisa outros sintomas além dos referidos acima, com por exemplo: dispneia, fadiga e mialgia. O índice de pneumonia grave relatada entre as séries de casos estudados nesse mesmo estudo, variou de 0 a 14\%, com a prevalência de casos que necessitara de internação em unidade de terapia intensiva (UTI). O autor relata ainda alguns casos de aborto espontâneo. O mesmo foi observado por Liu et al. (2020b) que incluíram 13 gestantes positivas para o vírus SARS-CoV-2 em seu estudo na China e evidenciaram que a maior parte das mulheres referiram sintomas leves a moderados e uma precisou de assistência em UTI.

Diversas pesquisas evidenciam a preocupação quanto à exposição e risco de contágio das gestantes e seus bebês, no entanto ponderam o fato de que neste momento, o atendimento pré-natal e a realização de procedimentos não poderiam deixar de ser ofertados, uma vez que são fundamentais na avaliação da evolução da gestação e identificação de fatores de risco para morbimortalidade materno-fetal. Assim, é relevante frisar a necessidade de se observar com mais cautela a urgência dos procedimentos e do acompanhamento de forma presencial durante a gestação, tendo em vista que para casos como gravidez de risco, a parada total dos serviços de acompanham do pré-natal, pode resultar em risco negativo, tão quanto o próprio contágio em si (Abenfo/Sobep, 2020; Brasil, 2020a; Liang \& Acharya, 2020; Rcog, 2020; Unfpa, 2020).

Contudo, em casos de suspeita ou infecção confirmada, é necessário que consultas ou procedimentos previstos sejam reagendados para data em que o período de contágio tenha acabado. Porém, caso o atendimento seja inadiável, a consulta deve ocorrer em ambiente seguro para que não haja contaminação da equipe (Rcog, 2020; Brasil, 2020a). Outrossim, deve-se informar a gestante que em caso de sintomas suspeitos de coronavírus, esta deverá entrar em contato, de forma não presencial, com a equipe de saúde para esclarecer suas dúvidas, antes de dirigir-se até a unidade de saúde (Rondelli et al., 2020).

Nesse contexto, considerando os aspectos psíquicos das gestantes, Ferreira et al. (2020) enfatizam a importância de reclusão domiciliar de todos os membros da família da gestante durante a pandemia, por outro lado, esse isolamento social pode aumentar a ansiedade das gestantes, evoluindo até mesmo para depressão.

Considerando o impacto psicológico durante a pandemia, uma pesquisa realizada na China classificou seu potencial de moderado a grave em quase metade dos indivíduos participantes do estudo. Com relação ao nível de ansiedade, um terço dos entrevistados resultaram em transtorno entre moderado e grave (Wang et al., 2020).

Logo, a gestante que se encontra nesse contexto de isolamento social, tem os mesmos anseios, seja por suspeita ou por diagnóstico positivo para COVID-19. O que aumenta quando após testar positivo, esta não pode ter contato direto com seu filho, elevando assim o grau ansiedade, estresse, e em alguns casos, depressão pós-parto. Considerando ainda, que há inúmeras lacunas sobre a COVID-19 a ansiedade pode causar acentuação dos quadros psíquicos nessas gestantes (Jardim et al., 2019).

Além disso, ainda há outro fator preocupante e que gera estresse, tristeza e ansiedade nas puérperas com diagnóstico positivo para COVID-19, a amamentação, visto que a mulher não poderá ter contato direto com seu recém-nascido-RN, como medida de proteção, o que desencadeia aflições por não poder nutrir seu bebê como planejou durante a gestação (Paz et al., 2020).

Contudo, percebe-se que há algumas controvérsias com relação ao contato entre mãe com coronavírus e seu RN. Alguns autores, não recomendam esse contato entre as mães infectadas e bebês, por causa do risco de transmissão do vírus para o mesmo (Abenfo/Sobep, 2020; Rasmussen et al., 2020; Liang \& Acharya, 2020; Qiao 2020).

Sobretudo, a Sociedade Brasileira de Pediatria (2020) enfatiza que é permitido o contato entre mãe e RN, separandose estes, apenas em necessidade de cuidados de rotina com o bebê, bem como, considera aceitável a estadia da mãe no alojamento conjunto, desde que medidas de segurança sejam tomadas como precaução da disseminação do vírus. 
Já, com relação as particularidades obstétricas e perinatais da infecção por COVID-19, é relevante frisar que gestantes com alguma comorbidade, infectadas por Sars-Cov-2 que evoluem para um quadro grave, têm probabilidade elevada de necessitar de parto cesáreo de emergência ou de evoluir para parto prematuro, elevando o risco de morte, tanto materna, como neonatal (Li et al., 2020).

Mesmo diante de um cenário adverso, deve-se respeitar os direitos das gestantes, mesmo aquelas com diagnóstico de infecção pela COVID-19, contudo, algumas instituições hospitalares tem utilizado o isolamento durante o trabalho de parto como medida preventiva da infecção por COVID- 19, violando o direito da mulher de ter um acompanhante antes, durante e após o parto, conforme previsto na Lei no 11.108, de 07 de abril de 2005 (Brasil, 2005).

Sabe-se que no momento do parto, a presença de uma pessoa escolhida pela gestante é capaz de amenizar a dor, proporcionar segurança, bem-estar físico, emocional e mental durante o processo (Souza \& Gualda, 2016). Atentando-se para os benefícios de ter um acompanhante durante o trabalho de parto, é improtelável que medidas voltadas para a prevenção da transmissibilidade da COVID-19 durante o parto sejam formuladas, para que não reverberem em vivências negativas durante um momento tão especial para a mulher, como é o parto. Pelo exposto, é indispensável que os hospitais e maternidades identifiquem os direitos das gestantes a fim de garantir um trabalho de parto humanizado e seguro. Para tal, protocolos de paramentação como medidas de proteção podem ser adotados, bem como outras estratégias, de modo que o risco de infecção no momento do parto seja diminuído ou nulo, desta forma, assegurando os direitos das gestantes (Estrela et al., 2020).

No Brasil, o Ministério da Saúde (Brasil, 2020d), permite que um acompanhante esteja durante todo o trabalho de parto, ainda que a mulher seja diagnosticada com Sars-Cov-2, sobretudo, com as condições de não haver revezamentos ou troca de acompanhante e que o visitante não seja considerado grupo de risco para COVID-19.

Levando-se em consideração esse contexto, é fundamental o papel do enfermeiro tanto na Atenção Primária à Saúde (APS), durante a consulta do pré-natal ou puerperal, quanto na atenção hospitalar, para realizar orientações a gestante e seus familiares. Vale enfatizar que na APS, além dos cuidados que já são preconizados durante o pré-natal, deve-se incluir orientações sobre o coronavírus, a fim de esclarecer mitos e desmentir fake news, visando que medidas preventivas contra a COVID-19 sejam mais efetivas. Deve-se orientar sobre a frequência e a técnica correta de higienização das mãos, bem como das superfícies de contato, sobre a importância do distanciamento social e do uso máscaras, cuidados estes que devem ser seguidos tanto no domicílio da gestante como em locais com muitas pessoas, como é o caso da sala de espera dos hospitais e da unidade básica de saúde (Estrela et al., 2020).

\section{Considerações Finais}

Diante da pesquisa realizada, percebe-se os grandes desafios e lacunas que existem no cenário pandêmico. Muitas dessas incertezas, incluem os cuidados com as gestantes e como proceder diante de um trabalho de parto de uma mãe diagnosticada com COVID-19. Existem controvérsias entre alguns autores, enquanto uns afirmam que não é possível permitir que a gestante dê à luz ao lado de um acompanhante, como medida de prevenção do coronavírus, outros, afirmam que os direitos das gestantes não podem ser infringidos.

Com relação ao contato da mãe infectada com Coronavírus e seu RN, também existe um conflito nas evidências encontradas. Alguns pesquisadores consideram perigoso para o bebê, enquanto outros, defendem o contato direto e constante entre os mesmos.

Com relação a ansiedade, estresse e depressão, os pesquisadores da amostra foram unânimes em relacionar o isolamento social causado pela pandemia, como um fator desencadeante de tais problemas psicológicos em gestantes.

Já, as complicações gestacionais causadas pela COVID-19 ainda estão sendo esclarecidas, a medida em que vão surgindo novos casos e estudos. Outro ponto polêmico entre os autores, enquanto uns relatam que as gestantes e crianças estão 
isentas de complicações, outros estudos afirmam que as grávidas e seus RNs devem fazer parte do grupo de riscos, devido suas condições metabólicas e imunológicas. O que se pode perceber, é que os sintomas e gravidade tem variação, algumas gestantes infectadas sentiram apenas febre baixa e tosse, enquanto outras, foram diagnosticadas com pneumonia grave e até mesmo disfunção de órgãos vitais.

No mais, enfatiza-se a importância dos profissionais da saúde, no tocante as orientações prestadas a gestante e seus familiares, como forma de prevenir a infecção, desmitificar mitos, esclarecendo dúvidas que possam contribuir para diminuir a sobrecarga de ansiedade durante gestação.

Pelo exposto, é de extrema importância que novas pesquisas que relacionem a COVID-19 entre as gestantes, sejam realizadas, para que assim, sejam esclarecidas as dúvidas e controvérsias que pairam no meio cientifico no tocante a temática.

\section{Referências}

Associação brasileira de enfermeiros obstetras e obstetrizes. Sociedade Brasileira de Enfeiras Pediatras. Nota técnica referente aos cuidados da equipe de enfermagem obstétrica, neonatal e pediátrica diante de caso suspeito ou confirmado. Abril, 2020.

Bastos, L. F. C. S. (2020). OPAS/OMS Brasil-Folha informativa-COVID-19 (doença causada pelo novo coronavírus). OPAS/OMS. https://www. paho. org/bra/index. php.

Brasil, Ministério da Saúde. Lei n ${ }^{\circ} 11.108$, de 07 de abril de 2005. Dispõe sobre acompanhamento durante o trabalho de parto, parto e pós-parto imediato. http://www.planalto.gov.br/ccivil_03/_ato2004-2006/2005/lei/111108.htm

Brasil. Ministério da Saúde. (2011). Secretaria de Atenção à Saúde. Departamento de Ações Programáticas Estratégicas. Portaria no 1.459, de 24 de junho de 2011. Institui, no âmbito do Sistema Único de Saúde - SUS - a Rede Cegonha. Brasília: Ministério da Saúde.

Brasil, Ministério da Saúde. (2020a). Secretaria de Atenção Primária a Saúde. Nota Técnica no 6 - Atenção às gestantes no contexto da infecção SARS-CoV-2. Ministério da Saúde: Brasília.

Brasil, Ministério da Saúde. (2020b). Secretaria de Atenção Primária a Saúde. Nota Técnica no 7 - Preservação da amamentação em situação de risco iminente de transmissão de SARS-CoV-2. Ministério da Saúde: Brasília.

Brasil, Ministério da Saúde. (2020c). Secretaria de Atenção Especializada à Saúde. Protocolo de manejo clínico da Covid-19 na Atenção Especializada. Ministério da Saúde: Brasília, 48 p.

Brasil, (2020d). Ministério da Saúde. Fluxo de manejo clínico de gestantes na atenção especializada. Eletrônica. https://www.saude.ms.gov.br/wpcontent/uploads/2020/03/Fluxo-de-manejo-clinico-de-gestantes.pdf.

Clode, N., Areia, A. L. (2020). Pregnant woman in the COVID-19 pandemia. Acta Obstet Ginecol Port, 14(3).

Estrela, F et al. (2020). Gestantes no contexto da pandemia da Covid-19: reflexões e desafios. Physis: Revista de Saúde Coletiva, 30(2).

Ferreira, M. J., Irigoyen, M. C., Consolim-Colombo, F., Saraiva, J. F. K., Angelis, K. (2020). Vida Fisicamente Ativa como Medida de Enfrentamento ao COVID-19. Arq Bras Cardiol. AHEAD, 114 (4).

Furlan, M. C. R et al. (2020). Gravidez e infecção por Coronavírus: desfechos maternos, fetais e neonatais - Revisão sistemática. Rev Cuid, Bucaramanga, 11(2).

Juan, J., Gil, M. M., Rong, Z., Zhang Y., Yang H., Poon L. C. (2020). Effect of coronavirus disease 2019 (COVID-19) on maternal, perinatal and neonatal outcome: systematic review. Ultrasound in Obstetrics \& Gynecology, 56, 15-27.

Jardim, T., Viana, G. P., Cruz, W. O., Assis, T. O., Lemos, G. D., Almeida, K. J. S., Maia, C. S., Lemos-Jordão, A. J. J. M. (2019). Principais fatores relacionados à impossibilidade de amamentação em Puérperas assistidas no Isea. Braz. J Health Rev, 2 (6).

Li, N. et al. (2020). Maternal and neonatal outcomes of pregnant women with COVID-19 pneumonia: a case-control study. MedRxiv, 3(2).

Liang, H., Acharya, G. (2020). Novel corona virus disease (COVID-19) in pregnancy: What clinical 0. recommendations to follow? Acta Obstetricia et Gynecologica Scandinavica, 99(4).

Liu, W. et al. (2020a). Coronavirus disease 2019 (COVID-19) during pregnancy: a case series. Preprints.

Liu, Y., Chen, H., Tang, K., Guo, Y. (2020b). Clinical manifestations and outcome of SARS-CoV-2 infection during pregnancy. Journal of Infection, 1-6.

Muhidin, S., Moghadam, Z. B., Vizheh, M. (2020). Analysis of Maternal Coronavirus Infections and Neonates Born to Mothers with 2019-nCoV; a Systematic Review. Arch Acad Emerg Med, 8 (1).

Paz, M. M. S. et al. (2020). Barriers imposed in the relationship between puerperal women and newborns in the COVID-19 pandemic scenario. Manuscript ID: RBSMI-2020-0231.

Qiao, J. (2020). What are the risks of COVID-19 infection in pregnant women? The Lancet, 395(10226). 
Rasmussen, S. A., Smulian, J. C., Lednicky, J. A., Wen, T. S., Jamieson, D. J. (2020). Coronavirus Disease 2019 (COVID-19) and Pregnancy: what obstetricians need to know. American Journal of Obstetrics and Gynecology, 1(12).

Rondelli, G. P. H. et al. (2020). Assistência às gestantes e recém-nascidos no contexto da infecção COVID-19: uma revisão sistemática. Revista Desafios, 7(1).

Rcog, Royal College Of Obstetricians \& Gynaecologistis. (2020). Coronavirus (COVID-19). Infection in Pregnancy, 2(3).

Santos, C. M. C., Pimenta, C. A. M., Nobre, M. R. C. (2007). A estratégia pico para a construção da pergunta de pesquisa e busca de evidências. Revista Latino-Americana de Enfermagem, 15 (3).

Sociedade Brasileira de Pediatria. (2020a). Recomendações para Assistência ao Recém-nascido na sala de parto de mãe com COVID-19 suspeita ou confirmada. Abril. https://www.sbp.com.br/imprensa/detalhe/nid/recomendacoes-para-assistencia-ao-recem-nascido-na-sala-de-parto-de-mae-com-covid-19suspeita-ou-confirmada/.

Souto, S. A., et al. (2020). Fear of childbirth in time of the new coronavirus pandemic. Rev Bras Enferm, 73(2).

Sousa, H. M. (2020). Comparação de dados sobre óbitos por Covid-19 entre três fontes de informação, Maranhão. Research, Society and Development, 9(9).

Souza, R. R. K., Gualda, D. M. R. (2016). A experiência da mulher e de seu acompanhante no parto em uma maternidade. Texto Contexto Enfer, 24(1).

Unfpa, United Nations Population Fund. (2020). Sexual and Reproductive Health and Rights, Maternal and Newborn Health \& COVID-19: Coronavirus Disease (COVID-19) Preparedness and Response UNFPA Interim Technical Brief. 2020.

Wagner, A et al. (2020). Vulnerabilidades para gestantes e puérperas durante a pandemia da COVID-19 no estado de Santa Catarina, Brasil. Hygeia-Revista Brasileira de Geografia Médica e da Saúde, 2(3).

Wang, C., Pan, R., Wan, X., Tan, Y., Xu, L., Ho, C. S., Ho, R. C. (2020). Immediate psychological responses and associated factors during the initial stage of the 2019 coronavirus disease (COVID-19) epidemic among the general population in China. Int J Environ Res Public Health, 17(5).

Zaigham, M., Andersson, O. (2020). Maternal and Perinatal Outcomes with Covid-19: a systematic review of 108 pregnancies. Acta Obstetricia Et Gynecologica Scandinavica, 7(1).

Zhu, H., Wang, L., Fang, C., Peng, S., Zhang, L., Chang, G., Xia, S., Zhou, W. (2020). Clinical analysis of 10 neonates born to mothers with 2019-nCoV pneumonia. Transl Pediatr, 9(1). 